\title{
SOBRE LA SUBJETIVIDAD DEL DICCIONARIO DE USO DEL ESPAÑOL DE MARÍA MOLINER
}

Escribimos estas líneas durante el año en que se conmemora, en Aragón especialmente, el centenario de María Moliner, nacida el 30 de marzo de 1900 en la localidad zaragozana de Paniza. Con esta pequeña aportación nos proponemos contribuir al conjunto de trabajos que se han acercado a su trayectoria vital y profesional con motivo del acontecimiento mencionado. Parece lógico, en este marco, que la obra que le otorgó merecida relevancia pública, el Diccionario de uso del español (Madrid, Gredos, 1966-67; en adelante DUE) haya acaparado buena parte de la atención dispensada a su figura ${ }^{1}$. La misma María Moliner llegó a declarar en alguna ocasión que el diccionario representaba el único mérito de su trayectoria profesional ${ }^{2}$, afirmación comprensible por la modestia que, es sabido, la caracterizó y quizá, también, por el deseo de dejar atrás la amarga experiencia bélica y el truncamiento, con ella, de las empresas culturales en las que participó con entusiasmo durante la Segunda República española.

Aunque todavía es reducido el número de monografías consagradas a desentrañar el enorme potencial informativo y la composición interna del $D U E^{3}$, las investigaciones y reseñas disponibles han permitido ya pasar revista a lo que ha sido la recepción del diccionario de María Moliner, entre la critica especializada y en el mundo de la cultura en general ${ }^{4}$. Puede concluirse al respecto que, en los años transcurridos desde su aparición, el DUE ha gozado de mejor acogida entre los creadores literarios y periodistas, por ejemplo, que entre los lingüistas. No obstante, las valoraciones vertidas sobre la obra por unos y otros presentan un tono muy dispar, que llega a ser contradictorio cuando se trata de aquilatar algunos aspectos del repertorio tales como su orientación descriptiva o normativa ${ }^{5}$. Ello no impide que exista un acuerdo amplio al juzgar otros componentes. Así ocurre a la hora de aplaudir la regeneración de la práctica definitoria llevada a cabo en el $D U E$ o, en sentido contrario, a la hora de enjuiciar negativamente tanto la ausencia casi total de información sobre la categoría gramatical de las entradas, como la presencia en la macroestructura de la terminología de zoología y botánica. Al margen de estos dos últimos detalles - de escasa entidad por ser fácilmente modificables ${ }^{6}$ - la valoración desfavorable del $D U E$ resulta también, si no unánime, generalizada y mucho más discutible, como veremos, cuando se evalúa el resto de sus materiales innovadores. Parece como si la tantas veces reclamada renovación de métodos y criterios en la confección de diccionarios de lengua -renovación de la que tan necesitada sigue estando la lexicografía española - contara con no pocos focos de resistencia, aferrados de forma consciente o no a las pautas marcadas por la tradición lexicográfica ortodoxa ${ }^{7}$.

Del conjunto de observaciones realizadas hasta la fecha sobre las opciones lexicográficas adoptadas por Maria Moliner, llama poderosamente la atención una insistente serie de críticas -difícil de documentar en las reseñas de otras obras lexicográficas- cuya reitera- 
ción parece encontrar una explicación en la coincidencia de varios autores en el análisis del diccionario $o$, más probablemente, en la repetición de un juicio de valor convertido en lugar común. En cualquier caso, en aquéllas se adivinan motivaciones ajenas, en alguna medida, al análisis lexicográfico. Al parecer, según las opiniones aludidas, uno de los rasgos más sobresalientes del $D U E$-el más destacado para algunos- vendría dado por el carácter predominantemente subjetivo e intuitivo del texto molineriano (o de alguno de sus elementos), condición cuya positividad es defendida en algunas -escasas-ocasiones, como ocurre en ciertos comentarios sobre el conjunto de la obra ${ }^{8}$. Así, para el escritor Francisco Umbral, «lo que tiene de personal, de poético, de fascinante [el DUE] es lo que tiene de intuitivo -óptica femenina del mundo- [...] $\aleph^{9}$. En el mismo sentido se manifiesta Manuel Seco, según el cual, en el $D U E$, «la intuición y la tenacidad tuvieron que llenar el vacío de una tradición previa que hubiera allanado el camino» ${ }^{10}$. La etiqueta se desliza incluso al celebrar «la revisión de las definiciones tradicionales y el valor intuitivo de las nuevas, la evitación de tautologías o círculos viciosos»" ${ }^{11}$, a pesar del método logicista -pormenorizado en los preliminares del $D U E-$ con que procede Moliner en la técnica definitoria. Pero si puede calificarse de desafortunado el empleo con intención laudatoria de la citada interpretación -ya que parece difícil conciliar el concepto de diccionario con una acepción positiva de intuiciónmenos convincente resulta todavia su uso como reprobación, al menos para referirse concretamente al $D U E^{12}$.

No obstante, los autores insisten con especial ahínco en la otra característica indicada (la subjetividad). Por ejemplo, las fuentes documentales empleadas -la selección del corpus- han sido objeto de múltiples críticas en este sentido. Así, Jesús Pena Seijas señaló en su día que "la inclusión de voces y de acepciones se rige por un criterio selectivo, no por un criterio científico, objetivo, comprobador de los hechos lingüisticos tal como aparecen» ${ }^{13}$. Manuel Seco precisa, en sintonía con lo anterior, que la obra «está construida tomando como casi únicas bases documentales la personal competencia hablante de la autora y -paradójicamente- el mismo Diccionario académico que se trataba de superar; con lo cual los criterios subjetivos priman más de lo conveniente sobre la información objetiva» ${ }^{14}$. Por su parte, Miguel Casas, haciéndose eco de la opinión expresada por Seco y Pena Seijas, insiste en que «esta obra es fruto de la norma de uso lingüistico de una sola persona y, por consiguiente, se rige por criterios subjetivos y selectivos» ${ }^{15}$.

Los aspectos menos apreciados -y más novedosos- del DUE no escapan, como cabía esperar, a críticas del mismo tenor. Así, la doble ordenación de entradas -alfabética y por familias relacionadas etimológicamente- suprimida en la edición de 1998, «suponía una cierta dosis de subjetividad", a juicio de José Álvaro Porto Dapena ${ }^{16}$. Todavía con mayor énfasis se insiste en la falta de objetividad que subyace a la distinción tipográfica entre palabras y acepciones usuales y no usuales. En este punto, Julio Fernández Sevilla echa de menos el manejo de procedimientos científicos y afirma con severidad que «se trata de estimaciones subjetivas que pueden inducir a error al lector confiado. Basta abrir el Diccionario para comprobar la arbitrariedad de tal decisión» ${ }^{17}$. A Manuel Seco le parece valiosa la diferenciación pero, al mismo tiempo, la despoja de toda validez al objetarle «que ha sido realizada siguiendo criterios exclusivamente subjetivos ${ }^{18}$. Finalmente, Miguel Casas, al comentar las observa- 
ciones citadas, sentencia lo siguiente: «Se impone, en última instancia, la norma selectiva de la autora y el criterio subjetivo en la cuantificación del porcentaje establecido» ${ }^{19}$.

Otros varios componentes del $D U E$ se hacen acreedores de similares manifestaciones. La ordenación de las distintas informaciones en el interior de los catálogos de palabras afines se rigen, en opinión de José Álvaro Porto Dapena, por un "criterio relativamente subjetivo» ${ }^{20}$. Y las pautas seguidas por María Moliner para escoger el artículo en el que insertar un catalogo ponen de relieve «claramente la subjetividad en la elaboración del diccionario», según Miguel Casas, quien poco después insiste en que «el criterio utilizado es subjetivo (competencia lingüística de la autora) y, para subsanarlo, haria falta la incorporación de métodos tanto estadísticos de frecuencias léxicas como sociolingüísticos, que describieran los usos reales del español ${ }^{21}$. La información sobre las relaciones antonímicas en el $D U E$ es juzgada con notable rigidez, desde presupuestos semánticos estructuralistas, por «la forma asistemática $y$ subjetiva con que está tratada ${ }^{22}$. Y en un artículo monográfico sobre la antonimia léxica en el repertorio de Moliner, Carmen Varo Varo titula significativamente uno de los apartados, "Tratamiento subjetivo de la antonimia» ${ }^{23}$, e insiste más adelante en que, en este aspecto, el criterio de la lexicógrafa aragonesa "no puede ser más subjetivo»" ${ }^{24}$. No corre mejor suerte la evaluación de las indicaciones o marcas de uso aportadas por Maria Moliner, que «se deben no a criterios lingüísticos sino a actitudes imposibles de aplicar en un análisis científico» ${ }^{25}$.

La coincidencia en el juicio resulta extraordinaria y casi abrumadora. La relevancia del tema reside en que las apreciaciones arriba acumuladas, nada habituales en las reseñas de otros diccionarios firmados por autor único, pueden estimarse no sólo poco acertadas en su atribución al $D U E$ sino, en buena medida, vacías de contenido. Cabe preguntarse, en una primera aproximación, si tales valoraciones se ajustan a la idea que poseía María Moliner sobre la subjetividad y la intuición. En la definición del adjetivo subjetivo-subjetiva en la obra que nos ocupa se leen las siguientes acepciones:

(1) (adj.). Se aplica, por oposición a «objetivo», a lo que se refiere al sujeto que piensa, siente, etc., y no a lo exterior a la mente de él.

(2) «Personal». Se dice de lo que depende de cada sujeto y no es igual para todos. (.) Se aplica, por oposición a «objetivo» o «imparcial», a los juicios, apreciaciones, etc., en que influyen la manera de ser o los sentimientos del que los hace, asi como a las personas cuyos juicios y apreciaciones son así.

Y en la entrada intuición encontramos tres acepciones, aparte de otra propia del lenguaje de la teología, de la que ahora prescindimos:

(1) Acto intelectivo que proporciona el conocimiento de las cosas por su sola percepción, sin razonamientos.

(2) Facultad de intuir.

(3) Facilidad para intuir: 'Tiene mucha intuición para las matemáticas'. (•) En lenguaje corriente, facilidad para percatarse de las cosas al primer golpe de vista o para darse cuenta, sin necesidad de razonamientos o explicaciones, de cosas que no son patentes para todos. 
Si nos atenemos a las acepciones del primer término, no se comprende bien en qué pueda diferenciarse el DUE de cualquier otro diccionario. En otras palabras, no se trata de dilucidar si la obra de Moliner está impregnada de sus planteamientos personales, sino de que, sencillamente, no existe otra posibilidad si nos referimos a una creación humana. Por lo tanto, como categoría de análisis metalexicográfico, la propiedad de subjetivo carece de validez porque no es apta para discriminar los rasgos distintivos de cada diccionario ${ }^{26}$. Ahora bien, la última subacepción permite ser interpretada en términos de subjetividad guiada por una ideología concreta, de modo cercano a como Manuel Seco describió la práctica definitoria del lexicógrafo decimonónico Ramón Joaquín Domínguez ${ }^{27}$. Siendo patente el fundamento ideológico-cultural de todo repertorio lexicográfico, los análisis disponibles apuntan, precisamente, a un DUE que se comporta, en el último sentido apuntado, como el diccionario menos subjetivo de su época, peculiaridad en la que sigue aventajando netamente a obras publicadas en un contexto sociopolítico, como el actual, significativamente más propicio que el del período en que Moliner desarrolló su labor lexicográfica ${ }^{28}$.

En cuanto al carácter intuitivo del $D U E$, parece claro, a tenor de las acepciones reproducidas más arriba, el motivo por el que una descripción que sitúa el diccionario en las proximidades de lo irracional, no se deja interpretar con facilidad en sentido favorable. En cualquier caso, esgrimida como elogio o como censura, la presunta intuición subyacente al $D U E$ no resiste un mínimo análisis técnico: no abundan los diccionarios del español que, como el de Moliner, confiesen con detalle el proyecto del que derivan, es decir, las opciones explícitas y lexicográficamente razonadas que sustentan el texto diccionaristico. Nada más ajeno al $D U E$ que ese fondo de improvisación creativa que se le atribuye como mérito o como deficiencia, según los casos. Que el origen de todo ello guarda escasa relación con el análisis propiamente lexicográfico ya se sospecha en la referencia de Umbral a la «óptica femenina del mundo». El estereotipo de género sobrevuela de igual modo sobre la crítica más ceñida a las cuestiones técnicas. Existen indicios, por ejemplo, de que algunos comentarios de la obra han venido precedidos de un examen poco contrastado. Así parece haber ocurrido con el repetido reproche sobre el recato de María Moliner ante los vulgarismos y voces tabuizadas ${ }^{29}$. Antes bien, del estudio específico sobre esa parcela del léxico se deriva que «el $D U E[\ldots]$ es el diccionario general de su época que más vocablos de este tipo incorpora» ${ }^{30}$.

Una de las imputaciones de subjetividad más insistente -ya se ha visto- hace hincapié en la debilidad de las fuentes documentales del $D U E$. En concreto, en el hecho de que su único sustento parezca ser, además del diccionario académico, el idiolecto de su autora. Como en este punto no se distingue del resto de la lexicografía no académica que la precedió -y coincide con la mayor parte de los diccionarios posteriores- vuelven a suscitarse las dudas sobre el trasfondo de la cuota suplementaria de subjetividad apreciada en el $D U E$, casualmente el único diccionario elaborado por una mujer. Pese a todo, las circunstancias de elaboración de la obra han sido expuestas de forma muy simplificada. Es evidente que la lexicógrafa aragonesa no acopió sistemáticamente un corpus lingüistico como etapa previa a la redacción de su obra ${ }^{31}$. Sin embargo, su imitación del diccionario académico (DRAE) estuvo sometida a una criba de mucho mayor calado que el reconocido comúnmente a partir de lo que estimamos una interpretación poco acertada del apartado preliminar del $D U E$ 
referido a la formación de la macroestructura de la obra ${ }^{32}$. En primer lugar, María Moliner amplió para el español el concepto de unidad de tratamiento lexicográfico anticipándose a su planteamiento en la investigación teórica posterior. Así, las entradas del $D U E$ no están constituidas sólo por palabras, sino también por formas ausentes de la mayoria de diccionarios de lengua: unidades fraseológicas (expresiones latinas como in saécula saeculorum), unidades inferiores a la palabra (prefijos y sufijos españoles, latinos y griegos) y por otras entidades poco habituales en los diccionarios de lengua como sonidos o grupos de sonidos, onomatopeyas, siglas, símbolos químicos, terminología científica de flora y fauna y muchos nombres propios. Además, se cuentan por centenares las ocasiones en que la autora señala que tal voz o acepción no figura en el $D R A E^{33}$ o que se incorpora a pesar de haber sido suprimida en 1956 ( $18^{\text {a }}$ edición del $\left.D R A E\right)$ :

drástico, -a. [...] (2) (acep. no incluida en el D. R. A. E.). Quizás por influencia del inglés, se emplea modernamente esta palabra con el significado de "enérgico» 0 "radical»: 'El gobierno dictó medidas drásticas para atajar la revolución'.

prefabricado, -a. (neologismo, no incluido en el D. R. A. E.). Se aplica a las construcciones o elementos constructivos que ordinariamente se hacen sobre el terreno en el sitio en donde van emplazados cuando, en vez de eso, están previamente construidos: 'Barracas prefabricadas. Bloques prefabricados de hormigón'.

sangordilla. (Navarra). *Lagartija. (Este nombre figuraba en ediciones anteriores del D. R. A. E., pero ha sido suprimido en la de 1956).

Por otro lado, la minuciosa descomposición en acepciones y subacepciones a la que somete los significados y las variantes de contenido no puede imputarse razonablemente a la competencia lingüística de la autora en exclusiva. Existen, por el contrario, numerosas evidencias de que Maria Moliner tuvo presentes obras de consulta distintas de las citadas hasta la fecha ${ }^{34} \mathrm{y}$ de que permaneció siempre atenta a los usos lingüísticos novedosos de su tiempo: «Constantemente estoy viendo en los periódicos o en las novelas expresiones que anoto para incluirlas», declaraba en una entrevista de 1972, coincidiendo con la propuesta para su ingreso en la Real Academia Española ${ }^{35}$. Además, en un conjunto numeroso de artículos se refiere de modo genérico a las fuentes, escritas u orales, de las que se ha servido. Así ocurre en entradas como las siguientes (destacamos en negrita los fragmentos en que se menciona la procedencia de la información):

abominar. [...] (El D. R. A. E. no da más construcción que la transitiva. No está, pues, autorizada explícitamente, aunque es frecuente en lenguaje hablado y escrito, la construcción con «de»: 'Los que abominan de las costumbres modernas').

benefactor, -a. (A pesar de que el D. R. A. E. da esta palabra como ant., no es dificil encontrarla en escritos actuales). Bienhechor.

confraternizar. Aunque el D. R. A. E. no lo incluye, y sin duda por influencia de «fraternizar», se emplea este verbo corrientemente en vez de "confraternar", hasta el punto de que este último es desconocido para muchas personas.

demanda. (casi desusado en lenguaje corriente hablado, salvo en la frase «en demanda de»; se usa a veces en lenguaje escrito, especialmente comercial: 'Siento comunicarle que no me es posible acceder a su demanda') [...]. 
neum-. (neumat-, pneum-, pneumat-; a diferencia de otros casos de palabras con inicial liquida, en éste la escritura usual es la fonética, incluso en escritos técnicos).

oficial, -a. En los cuerpos administrativos del Estado, categoría intermedia entre la de auxiliar y la de jefe. (V. «empleo».) (.) Persona que la desempeña. (Fem., «oficiala»; aunque figura en el D. R. A. E. y su uso es lógico, no se emplea, ni siquiera en los textos oficiales, en los que se dice, por ejemplo: «nombrando oficial de primer grado a D.a...»).

No parece, pues, que el texto molineriano pueda describirse, sin despojarlo de todo mérito, como "fruto de la norma de uso lingüístico de una sola persona»-tal como se sostiene en una de las objeciones citadas- ni se ve, de ser así, qué interés podría atesorar el diccionario para la comunidad lingüística. Tampoco terminan de convencer las acusaciones de que son objeto la distinción de voces y acepciones usuales y no usuales, así como la profusión y diversidad de marcas sobre la consideración social y estilística de las palabras. Cabe preguntarse si, planteada la cuestión en esos términos, no adolece igualmente de subjetividad la indistinción lexicográfica de la frecuencia relativa de uso del vocabulario o la ausencia de marcas orientadoras de la adecuación pragmática del léxico, habida cuenta de la relevancia comunicativa que poseen tales advertencias para los hablantes. No se defiende aquí, claro está, un programa informativo para la lexicografía que carezca de cualquier fundamento empírico. Tan solo se pretende poner de relieve que, en estos aspectos, la actividad lexicográfica de María Moliner se inserta en la tradición diccionarística occidental, cuyos horizontes amplia indudablemente. Y, en esta tradición, la catalogación de los usos verbales de la comunidad se ha derivado de la observación no sistemática de la lengua y sólo en algunos casos muy recientes se ha visto vinculada con la lingüistica de corpus. En lo que afecta concretamente a las llamadas marcas pragmáticas o de uso, puede afirmarse sin reservas que no se dispone en la actualidad de una amplia descripción de la lengua -y es poco probable que se lleve a cabo en los próximos años-capaz de proporcionar una alternativa satisfactoria a una lexicografia condicionada forzosamente por los instrumentos disponibles, de los que se sirve con mayor o menor pericia. Algo parecido podría advertirse a propósito del capitulo referido al tratamiento de la antonimia y de otras relaciones léxicas en el $D U E$, materia en la que, no obstante, se reconoce «que no se le puede pedir a $\mathrm{M}^{\mathrm{a}}$ Moliner la realización de algo que todavía continúa siendo poco menos que un objetivo intangible en la semántica actual» ${ }^{36}$.

No cabe duda de que el $D U E$ exhibe un grado de complejidad tipográfica, estructural e informativa exigente con el usuario que desee obtener de su consulta el máximo provecho. Su texto requiere del lector una cierta sensibilidad lingüística y una colaboración más activa y participativa que la necesaria para la mayoría de los repertorios léxicos. Se obtiene a cambio una riqueza informativa extraordinaria cuyas claves se facilitan en las páginas que encabezan la obra, lugar en el que Moliner argumenta con detalle las opciones por las que se ha decantado. Algo en lo que no se excede, en cambio, la edición de 1998 del DUE, incrementada, según el prólogo, en unas ocho mil entradas (un diez por ciento) y veinticinco mil acepciones, de cuyas fuentes no se informa al usuario. Como tampoco se aportan puntos de referencia, ignoramos si esas cantidades representan un incremento suficiente del léxico descrito, tres décadas después de la primera edición. Se puede constatar, sin embargo, la 
ausencia de voces de uso actual como asociacionismo, aulario, balcanización 'división de un estado en otros más pequeños acompañada de enfrentamientos externos e internos', canterano-a 'jugador formado en la cantera de un equipo'; centroderecha; centroizquierda; cinturón 'vía de circunvalación que rodea una ciudad' o ejecutar 'abrir un programa informático'. Todo apunta, por otro lado, al mantenimiento en el $D U E$ de 1998 del caudal léxico ya existente en la primera edición, sin que conste la revisión y posible supresión del vocabulario anticuado, cuya presencia en la obra original fue objeto de algunas críticas.

A pesar de lo dicho hasta aquí, no ocultaremos que la factura del DUE de 1966-67 presenta facetas problemáticas o discutibles. Así ocurre, por ejemplo, con la confusa organización de algunos componentes del artículo lexicográfico y la sinonimia de ciertas abreviaturas y fórmulas (o su polisemia a veces). Es el caso, asimismo, de la doble ordenación de las entradas, trabadas entre si por un tipo de relaciones léxicas con una finalidad informativa bien concreta y más provechosa de lo admitido en ocasiones. Algo similar ocurre, finalmente, con los desarrollos gramaticales en el cuerpo de la obra, cuya presencia en la macroestructura se ha calificado, contrariamente a nuestra opinión, de superflua o injustificada. Estos y otros elementos del diccionario de Moliner demandan una investigación ajena, en la medida de lo posible, a los estereotipos de género que algunos análisis parecen rezumar. El $D U E$ es, que duda cabe, un diccionario personal, pero en un sentido positivo, en lo que tiene de innovador.

\section{Notas}

1 Especialmente para los aspectos biográficos más desconocidos sobre Maria Moliner puede consultarse el conjunto de articulos que, como homenaje a la lexicógrafa aragonesa, fueron publicados por la revista Trébede. Mensual Aragonés de Análisis, Opinión y Cultura ( ${ }^{\circ} 36$, marzo de 2000). La vida y la obra de Maria Moliner fueron, asimismo, objeto de reconocimiento por parte del Instituto Cervantes, que insertó en la página web del Centro Virtual Cervantes una serie de estudios sobre el Diccionario de uso del español (DUE) y una bibliografia, muy completa, relativa a las distintas facetas de Maria Moliner (http://cvc.cervantes.es/actcult/mmoliner/). Además, para acercarse al análisis del $D U E$ es fundamental la consulta del volumen colectivo Estudios sobre el Diccionario de uso del español de Maria Moliner (M. Casas Gómez e I. Penadés Martinez [coords.] y Mª Tadea Díaz [ed.], Cádiz, Universidad de Cádiz, 1998) que contiene también un extenso apartado bibliográfico.

2 En esos términos se recoge su testimonio en el artículo anónimo «Maria Moliner, autora del "Diccionario de uso del español", murió ayer, a los 81 años» que publicó El País el 23 de enero de 1981.

3 Nos referimos al editado en 1966-67, único debido a la lexicógrafa aragonesa, reimpreso en múltiples ocasiones. En la actualidad existen en el mercado otros tres productos firmados por Maria Moliner, como la versión en CD-ROM del texto original, aparecida en 1996 y reeditada en 2000 con mejoras informáticas pero con el mismo contenido. En 1998 se edita en papel la esperada segunda edición del DUE, debida al Seminario de Lexicografia de la Editorial Gredos. Esta edición -en cuya cubierta se sigue leyendo el nombre de Maria Moliner y el título original de la obra - ha venido acompañada de una polémica que ha acabado en los tribunales. En esta salida de la obra se ha acometido una actualización del léxico descrito -que era acuciante y que quizá ha resultado demasiado timidapero se han sacrificado algunos de los elementos más caracteristicos del repertorio originario y otras muchas informaciones a cambio de mejorar -notablemente, eso si- la legibilidad del texto. La Editorial defiende la legitimidad de los cambios introducidos que respetan, al parecer, las orientaciones con las que $\mathrm{M}^{\mathrm{a}}$ Moliner trabajó para una futura reedición. Lo cierto es que el contenido concreto de las directrices que, según la Editorial, dejó marcadas $\mathrm{M}^{\mathrm{a}}$ Moliner no ha sido hecho público por la casa editora. Ésta, sin embargo, invoca de nuevo dichas pautas, en varias ocasiones, en los preliminares de la «Edición abreviada por la Editorial Gredos» del DUE (Madrid, Gredos, 2000) 
obra donde los lazos de unión con el texto primitivo quedan ya francamente desdibujados. Todo ello invita a reflexionar sobre la conveniencia de alargar indefinidamente la autoria de Maria Moliner, aun en el caso de que se conozcan con alguna precisión las soluciones lexicográficas que tenía previsto adoptar. No pareceria descabellado, por ejemplo, hacer del nombre de María Moliner un patrimonio de la lexicografia española transformándolo en marca independiente de productos lexicográficos, tal como ha ocurrido históricamente en el ámbito francófono con Larousse y Robert o con Webster en Estados Unidos.

4 A este respecto son de particular interés los trabajos de $\mathbf{M}^{\mathbf{a}}$ Antonia Martín Zorraquino y de Miguel Casas Gómez, que encabezan el volumen de Estudios sobre el Diccionario de uso del español de María Moliner citado en la nota 1.

5 Véase Miguel Casas Gómez, «El Diccionario de uso del español de Maria Moliner», en Estudios sobre el Diccionario de uso del español de Maria Moliner, op. cit., p. 33, nota 11 y p. 35, nota 14. Los comentaristas también discreparon en su momento sobre otras cuestiones como, por ejemplo, la supresión en la ordenación alfabética del $D U E$ de la ch y la $l l$ como letras autónomas. Véase, al respecto, Ma Antonia Martín Zorraquino, «Una lexicógrafa aragonesa: Da Maria Moliner», en Estudios sobre el Diccionario de uso del español de Maria Moliner, op. cit., p. 21.

6 Con buen criterio, en la edición de 1998 se ha incorporado el dato de la categoria gramatical en todas las entradas y se han agrupado en un apéndice los términos científicos de plantas y animales.

7 Claro está que la reacción contra la novedad lexicográfica no pertenece en exclusiva al ámbito hispánico. No nos es posible dar cuenta aquí de las copiosas críticas de que fueron objeto en su momento textos lexicográficos tan sugestivos como, por ejemplo, el Dictionnaire du français contemporain (Paris, Larousse, 1966), Le Robert méthodique. Dictionnaire méthodique du français actuel (Paris, Robert, 1982) o el Webster's Third New International Dictionary of the English Language (Springfield, G\&C Merriam, 1961).

8 Es nuestra la cursiva con que se destacan algunos fragmentos y palabras en las citas textuales que se aportan a continuación.

9 Cf. F. Umbral, «De diccionarios y otras reclusiones», El País, 3 de febrero de 1981, p. 29.

10 Cf. M. Seco, "Apéndice A: María Moliner», Estudios de lexicografia española, Madrid, Paraninfo, 1987, p. 211.

11 Cf. M. Casas, art. cit., p. 36.

12 La atribución al $D U E$ de la misma característica -la intuición- es vista por otros autores en un sentido opuesto, como apunta $\mathrm{M}^{\mathrm{a}}$ Antonia Martin Zorraquino: «En realidad las palabras de Umbral suponian una réplica a las que habia pronunciado unos dias antes Violeta Demonte, que, destacando la originalidad de la obra de Maria Moliner en cuanto intento de superación de los diccionarios clásicos, había censurado la fundamentación de algunos de sus resultados porque se asentaban en la intuición» (art. cit., p. 18, nota 12).

13 Cf. J. Pena Seijas, «Ma Moliner, Diccionario de uso del español (2 vols.)», Verba, 2, 1975, p. 340.

14 Cf. M. Seco, art. cit., p. 210.

15 Cf. M. Casas, art. cit., p. 33.

16 Cf. J. Á. Porto Dapena, "Diccionario de uso del español», en http://cvc.cervantes.es/actcult/mmoliner/, 2000.

17 Cf. J. Fernández Sevilla, "Acerca de algunos aspectos de la información lexicográfica», Boletín de la Academia Puertorriqueña de la Lengua Española, 6 (2), 1978, p. 84.

18 Cf. M. Seco, «Medio siglo de lexicografia española (1930-1980)», Estudios de lexicografia española, Madrid, Paraninfo, 1987, p. 202.

19

Cf. M. Casas, art. cit., p. 38 , nota 20.

20 Cf. J. Á. Porto Dapena, art. cit.

21 Cf. M. Casas, art. cit., p. 50.

22 Cf. M. Casas, art. cit., p. 45.

23 Cf. C. Varo Varo, "La antonimia léxica en el Diccionario de uso del español de María Moliner», en M. Casas Gómez e I. Penadés Martínez [coords.] y Ma Tadea Diaz [ed.], op. cit., p. 195.

24 Cf. C. Varo Varo, art. cit., p. 197.

25 Cf. M. Casas, art. cit., p. 50, nota 47. M. Casas se hace eco aqui de la opinión sobre este aspecto del $D U E$ expresada por Ma J. Tejera.

26 Excepto, quizá, en el nivel metodológico, donde seria posible, con muchas salvedades, oponer los repertorios debidos a un solo autor (subjetivos) a otros elaborados según un método colegiado (intersubjetivos). En cualquier 
caso, en todo equipo lexicográfico suele existir, aparte de una especialización de tareas, un sistema de trabajo jerarquizado con un responsable último. Por otro lado, ni subjetividad ni intuición (o sus equivalentes en inglés) figuran entre la terminologia lexicográfica en el Diccionario de lexicografia práctica (J. Martínez de Sousa, Barcelona, Biblograf, 1995) o en el Dictionary of lexicography (R. R. K. Hartmann and G. James, London and New York, Routledge, 1998).

27 Cf. M. Seco, «La definición lexicográfica subjetiva: el Diccionario de Domínguez», Estudios de lexicografía española, Madrid, Paraninfo, 1987, pp. 165-177.

28 Cf. $\mathrm{M}^{\mathrm{a}}$ Ángeles Calero, "Diccionario, pensamiento colectivo e ideologia (o los peligros de definir)», en Ma N. Vila et alii (eds.), Asi son los diccionarios, Lleida, Universitat de Lleida, 1999, pp. 149-201.

29 El recato de Moliner es criticado por C. Smith, «M" Moliner, Diccionario de uso del español, vol. I, A-G, 1966, vol. II, H-Z, 1967», Bulletin of Hispanic Studies, 46, 1969, p. 51; H. Schneider, "M" Moliner, Diccionario de uso del español, 1966-67, 2 Bände», Romanistisches Jahrbuch, 22, 1971, p. 377; G. Haensch et alii, La lexicografia. De la lingüistica teórica a la lexicografia práctica, Madrid, Gredos, 1982, p. 155; C. Pratt, «El Diccionario de la Academia y el María Moliner", Libros, 23, 1984, p. 6 y J. Martínez de Sousa, en J. A. Pérez Bouza, M. Casas y J. Martinez de Sousa "María Moliner a la luz de la lexicologia y lexicografia modernas», en C. Martín Vide (ed.), Actas del X Congreso de Lenguajes Naturales y Lenguajes Formales, Barcelona, PPU, pp. 665-666.

Cf. M. Casas, art. cit., p. 55.

31 Ahora bien, conviene no olvidar que, en el siglo veinte, sólo dos repertorios de lengua se han confeccionado a partir de esa tarea documental preliminar: el Diccionario del español actual (M. Seco, O. Andrés y G. Ramos, 2 vols., Madrid, Aguilar, 1999) y el Diccionario del español de México, dirigido por Luis Fernando Lara. Y sólo en este último -pendiente aún de ser editado en su versión definitiva- se han utilizado realmente mediciones lexicométricas cuyas virtudes han quedado de manifiesto, al igual que sus limitaciones, que han obligado a incorporar informaciones basadas en el conocimiento de la lengua del equipo lexicográfico y en otros criterios cualitativos no estadísticos. Cf. L. F. Lara et alii, Investigaciones lingüisticas en lexicografia, México D. F., El Colegio de México, 1979 y G. Haensch et alii, op. cit., pp. 395-451.

32 Desde otro punto de vista, se recoge una consideración similar en M. Casas, art. cit., p. 49.

33 Al margen quedan las admitidas por la Academia para la que luego fue la $19^{\mathrm{a}}$ edición del $D R A E$ en 1970 . En estos casos, Moliner introduce en el articulo un comentario como el del siguiente ejemplo: «escayolista. (La R. A. ha acordado su inclusión en el D. R. A. E.). Operario que hace molduras de escayola».

34 Además del DRAE, Moliner tuvo presentes el Diccionario ideológico de Julio Casares y Breve Diccionario Etimologico de Corominas. Suele olvidarse en esta nómina el Diccionario manual e ilustrado de la lengua española, publicado por la Academia, citado en numerosas ocasiones en las páginas del DUE. Además, la autora se auxilió, al menos, de la Enciclopedia Espasa (véase la voz fané) y de diccionarios de lenguas distintas del español, a los que se refiere genéricamente (véanse, por ejemplo, las entradas obuis y shah).

La entrevista fue publicada en el diario $A B C$ el 25 de junio de 1972.

36 Cr. M. Casas, art. cit., p. 37, nota 18. 


\section{MARÍE MOLINER}

Kritične ocene slovarja Marie Moliner nam precej dobro kažejo, kateri deli slovarja so cenjeni in kateri ne. Izmed negativnih kritik najbolj pritegne pozornost tista, ki nastanku slovarja očita določeno stopnjo subjektivnosti in intuicije. V našem prispevku, napisanem $v$ letu, ko obhajamo stoletnico rojstva Marie Moliner (rodila se je leta 1900 v Panizi, Zaragoza), želimo dokazati, da imajo argumenti navedenih kritik, iz katerih veje predsodek oziroma zadržanost do avtorice-ženske, trhle temelje. 